\title{
Stochastic resonance in a dipole
}

\author{
J. M. G. Vilar, A. Pérez-Madrid, and J. M. Rubí \\ Departament de Física Fonamental, Facultat de Física, Universitat de Barcelona, Diagonal 647, E-08028 Barcelona, Spain
}

(Received 30 May 1996)

\begin{abstract}
We show that the dipole, a system usually proposed to model relaxation phenomena, exhibits a maximum in the signal-to-noise ratio at a nonzero noise level, thus indicating the appearance of stochastic resonance. The phenomenon occurs in two different situations, i.e., when the minimum of the potential of the dipole remains fixed in time and when it switches periodically between two equilibrium points. We have also found that the signal-to-noise ratio has a maximum for a certain value of the amplitude of the oscillating field. [S1063-651X(96)07411-9]
\end{abstract}

PACS number(s): 05.40. $+\mathrm{j}, 41.90 .+\mathrm{e}, 82.70 . \mathrm{Dd}$

The phenomenon of stochastic resonance (SR) [1-10] may be characterized by the appearance of a maximum in the output signal-to-noise ratio (SNR) at an optimized nonzero noise level. Although early studies of SR were restricted to bistable systems, later developments have shown that this phenomenon is also present in a wider class of situations, including threshold devices and fire and reset models. In all these cases, a combination of the periodic input signal and the presence of an optimized noise may give rise to SR when the system crosses a barrier or a threshold in a coherent fashion. This phenomenon has been found in many particular realizations such as a Schmitt trigger [11], lasers [2], neurons $[12,13]$, and magnetic particles [14].

In spite of the efforts devoted to show the ubiquity of the phenomenon in many branches of physics, chemistry, and biology, there is an aspect that has not been considered up to now. It refers to the possibility of the appearance of a maximum in the SNR [15] during the relaxation process of a system towards a single minimum, due to the combined action of the noise and the periodic signal. In this paper we address this possibility precisely, which leads to different applications of SR in the domain of the relaxation phenomena. To be more specific, consider a system in an equilibrium state, stable or unstable, where no motion is observed. The addition of noise removes the system from this situation in such a way that a force field acts on it. If this force varies periodically in time, then SR may occur. As a physical realization of the phenomenon we have shown that SR may take place in a single dipole under a periodic external field.

Let us consider the relaxational dynamics of a single di-
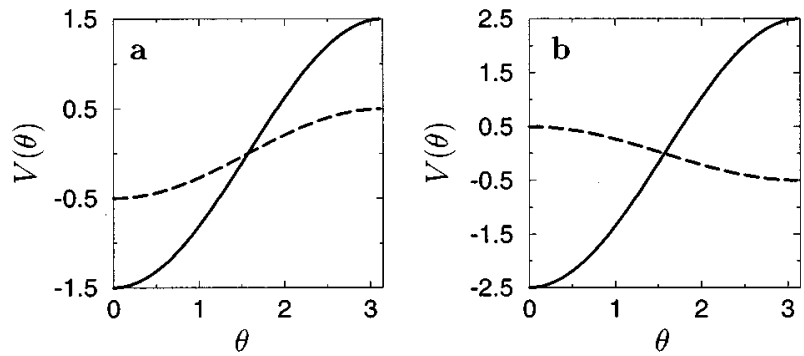

FIG. 1. Potential $V(\theta)=-h(t) \cos (\theta)$ for the maximum of $h(t)$ (continuous line) and for the minimum (dashed line), with $k=1$ and (a) $\alpha=0.5$, and (b) $\alpha=1.5$. pole $[16,17]$ in an external field, which may be described by the Langevin equation governing the dynamics of the unit vector $\vec{R}$ along the direction of the dipole moment

$$
\frac{d \hat{\vec{R}}}{d t}=[\vec{h}(t) \times \hat{\vec{R}}+\vec{\xi}(t)] \times \hat{\vec{R}}
$$

Here the total field $\vec{h}(t)=k\left[1+\alpha \sin \left(\omega_{0} t\right)\right] \hat{\vec{z}}$, with $\hat{\vec{z}}$ a unit vector, consists of a constant plus an oscillating field and it is characterized by the parameters $k$ and $\alpha$. The noise term $\vec{\xi}(t)$ may originate from the presence of an external random field or from thermal fluctuations. It is assumed to be Gaussian white noise with zero mean and second moment $\left\langle\xi_{i}(t) \xi_{j}(t+\tau)\right\rangle=D \delta_{i j} \delta(\tau)$, defining the noise level $D$.

The potential energy of the dipole is $V(\theta)=-h(t) \cos \theta$, where $\theta$ is the angle between its direction and the direction of the external field $[\cos (\theta)=\hat{\vec{z}} \cdot \hat{\vec{R}}]$. Two qualitatively different situations may occur, one for small $(\alpha<1)$ and the other for high $(\alpha>1)$ oscillating fields. In the first case, the potential has a minimum which remains fixed in time at $\theta=0$ [Fig. 1(a)]. In the absence of noise, once the dipole relaxes

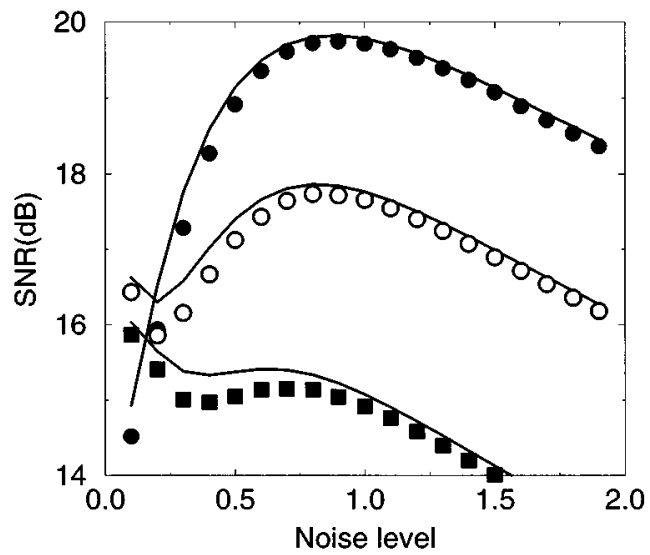

FIG. 2. SNR as a function of the noise level $D$, obtained through simulations (symbols) and computed from Eq. (6) (lines). The values of the parameters used here are $k=1, \omega_{0}=0.01, \alpha=0.5$ (squares), $\alpha=0.7$ (open circles), and $\alpha=0.9$ (filled circles). 

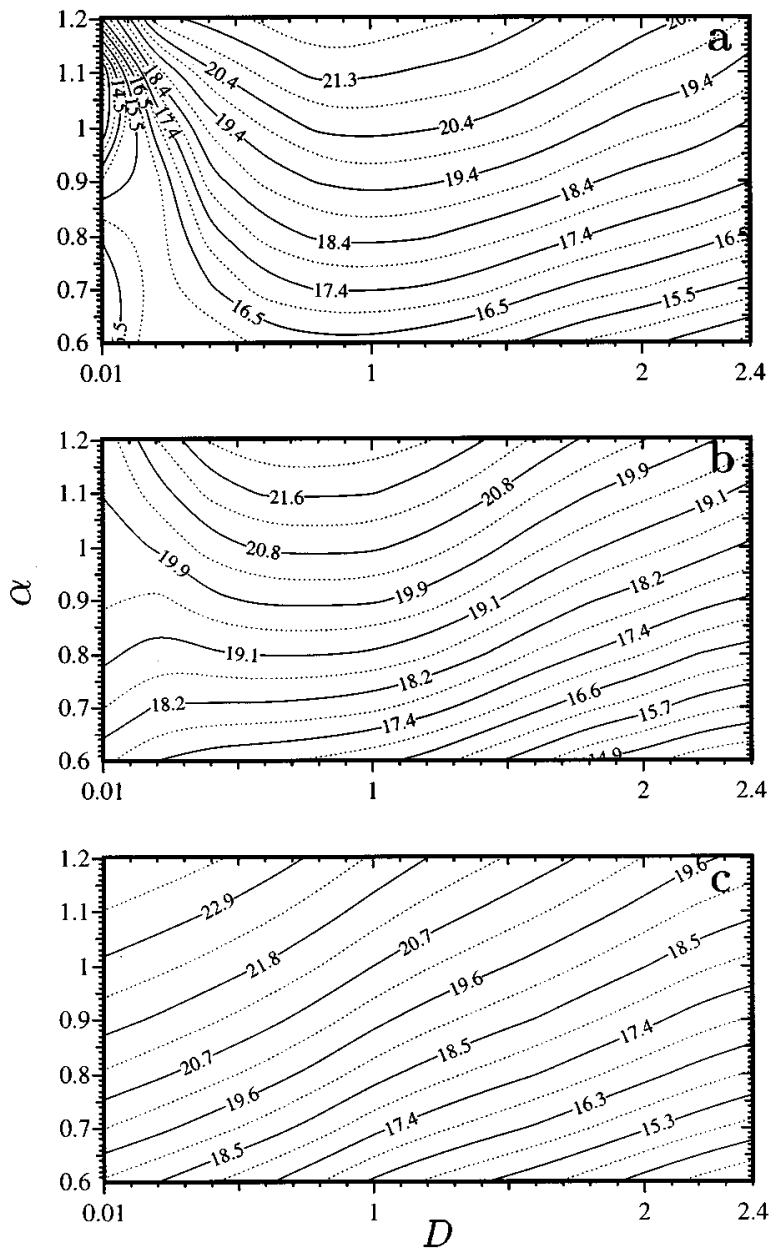

FIG. 3. Contour plot of the SNR as a function of both the noise level $D$ and the amplitude of the oscillating field $\alpha$, for $k=1$ and the frequencies (a) $\omega_{0} / 2 \pi=0.01$, (b) $\omega_{0} / 2 \pi=0.1$, and (c) $\omega_{0} / 2 \pi=1$.

toward this minimum no motion is observed, whereas when noise is added, the motion is modulated by the oscillating field. In the second case [Fig. 1(b)], the position of the minimum switches periodically in time between the equilibrium points $\theta=0$ and $\pi$. In the absence of noise, starting from any arbitrary initial condition $\left(\theta_{0} \neq \pi\right)$, the dipole relaxes towards $\theta=0$, in spite of this point becoming stable or unstable periodically. When a small amount of noise acts on the dipole, it may leave the unstable equilibrium point and move toward the stable one. We could easily infer that the output signal has a maximum as a function of $D$, but it is not a trivial matter to elucidate the behavior of the SNR, because both signal and noise go to zero as $D$ decreases.

Since we are concerned with the magnitude $\cos (\theta)$, which for a magnetic dipole corresponds to the component of the magnetization along the field, the averaged power spectrum is given by

$$
P(\omega) \equiv \int_{-\infty}^{\infty} d s e^{i \omega s} \int_{0}^{2 \pi / \omega_{0}}\left\langle\cos \left(\theta_{t+s}\right) \cos \left(\theta_{t}\right)\right\rangle d t .
$$

Analytical studies of the system, using linear-response theory (LRT), which is valid for small fields, show a maxi-

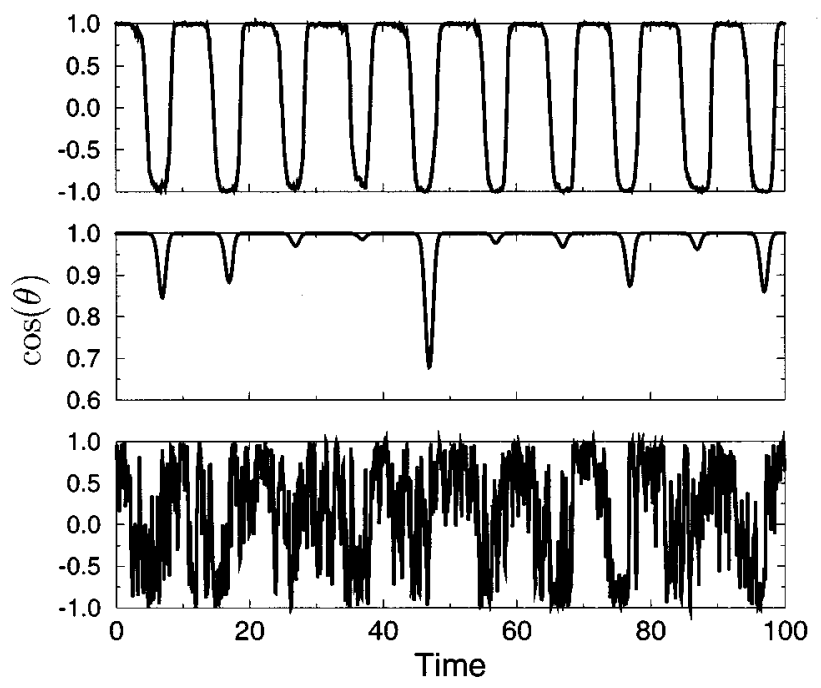

FIG. 4. Time evolution of $\cos (\theta)$ for $\alpha=3, k=1, \omega_{0} / 2 \pi=0.1$, and the noise levels: $D=3.3 \times 10^{-2}$ (top), $D=3.3 \times 10^{-6}$ (middle), and $D=3.3$ (bottom).

mum in the signal, i.e., in the susceptibility, as a function of the noise level $D$. However, the SNR is a monotonically decreasing function of $D$. One finds

$$
I_{\mathrm{SNR}}=L(\mu) k \alpha^{2}
$$

obtained from the fluctuation-dissipation theorem [18] and the expression for the complex susceptibility [19]

$$
\chi(\omega)=\frac{\frac{\mu}{k} L^{\prime}(\mu)}{1+i \omega \tau(\mu)}
$$

where $\tau(\mu)=\mu^{2} L^{\prime}(\mu) / 2 k L(\mu)$ is the relaxation time and $L(\mu)=\operatorname{coth}(\mu)-1 / \mu$ is the Langevin function, with $\mu \equiv 2 h(t) / D$.

Beyond the domain of applicability of LRT, we have found a range of values of the parameters for which the SNR exhibits a maximum for a nonzero noise level. Our result is obtained from numerical simulations by integrating the corresponding Langevin equation by means of a standard second-order Runge-Kutta method for stochastic differential equations $[20,21]$. The SNR, computed numerically through the averaged power spectrum, is defined as

$$
I_{\mathrm{SNR}}=10 \log _{10} \frac{A\left(\omega_{0}\right)}{N\left(\omega_{0}\right)}
$$

where $A\left(\omega_{0}\right)$ is the area of the peak above the noise floor and $N\left(\omega_{0}\right)$ is the noise background at the frequency $\omega_{0}$. In Fig. 2 we have depicted the SNR for finite amplitudes of the oscillating field. We have observed that for small $\alpha$ the SNR is a monotonically decreasing function of the noise, as predicted by LRT. Nevertheless, for higher values of $\alpha$ the SNR has a maximum at $D \neq 0$ (Fig. 2). When the amplitude decreases, this maximum becomes less sharp and disappears at lower $\alpha$ in a continuous fashion.

This behavior could also be obtained analytically in the limit of small frequencies, i.e., when the system relaxes 


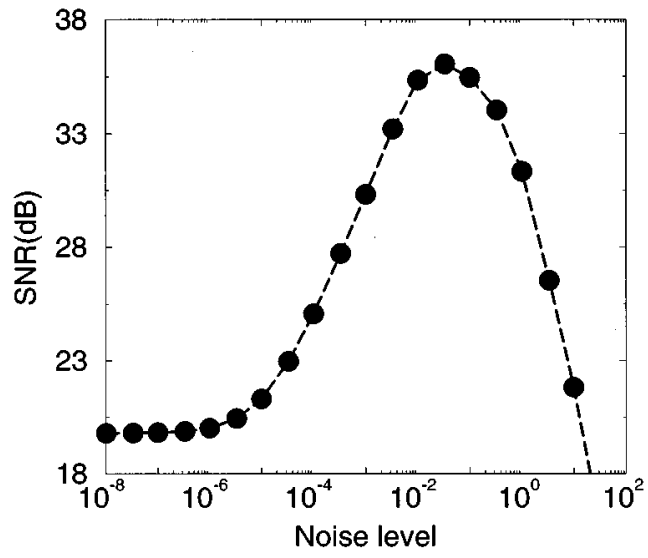

FIG. 5. SNR for $\alpha=3, k=1$, and $\omega_{0} / 2 \pi=0.1$.

faster than the period of the external field. The power spectrum may then be computed from the approximated expression for the correlation function

$$
\left\langle\cos \left(\theta_{t}\right) \cos \left(\theta_{t^{\prime}}\right)\right\rangle \approx L_{t} L_{t^{\prime}}\left(1-e^{\lambda_{t, t^{\prime}}}\right)+\left(1-2 \frac{L_{t}}{\mu_{t}}\right) e^{\lambda_{t, t^{\prime}}}
$$

where $\quad L_{t} \equiv L(\mu(t)), \quad \lambda_{t, t^{\prime}} \equiv-\int_{t}^{t^{\prime}} \tau(\mu(s))^{-1} d s, \quad$ and $\mu_{t}$ $\equiv \mu(t)$. Additionally, to obtain Eq. (6) we have used the previous analytical expression of the relaxation time, which differs from the exact value only by a few percent [19]. The results obtained by using Eq. (6) in Eq. (2) are depicted in Fig. 2. It is interesting to notice that the SNR defined from Eq. (2), which has dimensions of the inverse of time, has been written in dimensionless units in such a way that it coincides with the definition of Eq. (5). The small differences between the analytical and simulation results occur at intermediate values of $D$ and come from the approximation in the relaxation time.

In the previous results we have shown a dependence of the appearance of SR on the amplitude of the input signal. This dependence contrasts with the results obtained for the bistable quartic potential, in which the maximum of the SNR disappears for sufficiently large amplitudes of the oscillating field. Additionally, numerical simulations show a qualitative change of the SNR upon varying the frequency of the signal $\omega_{0}$. The results obtained indicate that when increasing $\omega_{0}$, for a fixed $\alpha$, the maximum of the SNR disappears, as illustrated in Fig. 3.

Although in Fig. 3 we have depicted the SNR for some values of $\alpha>1$, this behavior is similar to that for $\alpha<1$ because when the dipole is able to leave the neighborhood of $\theta=0$ the noise is so high that it destroys its coherent motion. However, fixed $\omega_{0}$, for large enough $\alpha$, the amount of noise necessary in order for the dipole to leave the unstable equilibrium point, does not destroy its coherent motion. This behavior is illustrated in Fig. 4 for $\alpha=3, k=1$, and $\omega_{0}=0.1$ and is similar to the one found in [22]. It shows the time evolution of $\cos (\theta)$ for the optimal, lower, and greater noise levels. For the previous values of the parameters, the SNR (Fig. 5) exhibits a maximum at $D=0.033$. The addition of

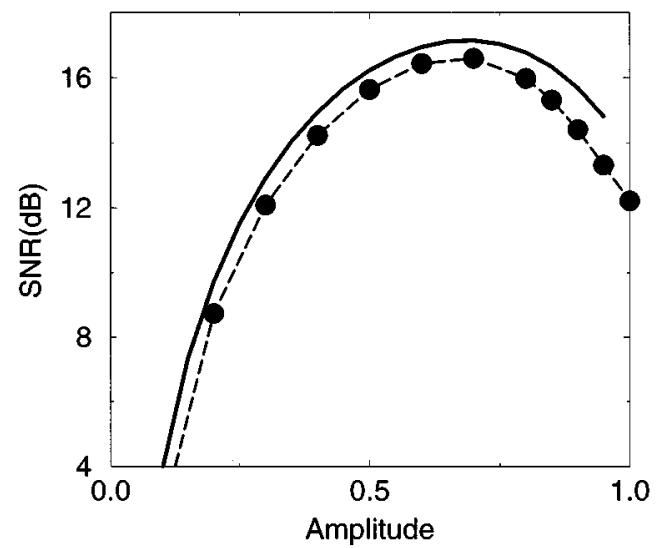

FIG. 6. SNR as a function of amplitude of the oscillating field $\alpha$, for $k=1, \omega_{0}=0.01$, and $D=0.01$. Obtained through simulations (symbols) and computed from Eq. (7) (solid line).

noise then increases the SNR up to $15 \mathrm{~dB}$ over its value at the limit of $D$ going to zero. For the optimal value of $D$ the behavior of the dipole looks surprisingly similar to that for the bistable potential.

Turning our attention to Fig. 3(a), we note another even more interesting feature. For low $D$ the SNR is not a monotonically increasing function of the amplitude. This kind of behavior implies that in some range of values of the parameters, a small signal is more easily detected than a greater one. The output signal, however, is always an increasing function of the amplitude. The maximum of the SNR (Fig. 6 ), as a function of $\alpha$, is due to the noise at the frequency $\omega_{0}$ increasing faster than the signal when $\alpha$ approaches to 1. For $D=0.01$ and $\omega_{0}=0.01$ the SNR has a maximum at $\alpha \approx 0.7$. In such a case, the SNR may be evaluated by approximating the dynamics around the minimum by a twodimensional Ornstein-Uhlenbeck process [23]. When this approximation holds the correlation function reads

$$
\left\langle\cos \left(\theta_{t+\tau}\right) \cos \left(\theta_{t}\right)\right\rangle \approx D^{2} \frac{1+\exp \left(\int_{t}^{t+\tau} h(s) d s\right)}{2 h(t) h(t+\tau)} .
$$

The SNR has been computed as in Eq. (6). The corresponding result is represented in Fig. 6. In spite of the approximation made to obtain Eq. (7), this result is in excellent agreement with the one obtained through simulations for the range where this approximation makes sense. It must be pointed out that the SNR, for low amplitudes, follows the quadratic law, $I_{\mathrm{SNR}} \propto \alpha^{2}$. Note that the result given by Eq. (7) corresponds to the one for a linear system, that shows that nonlinearities do not play any role in the appearance of this phenomenon. What is remarkable is the great deal of generality of this result, since it may hold for any system around a minimum.

In summary, we have shown the existence of SR in a dipole, a system which has been widely analyzed due to its importance as a simple relaxation model. It is well known that the susceptibility of the dipole exhibits a maximum at a certain value of the temperature (strength of the noise) [24]. In this paper we have also shown that a maximum also appears in the SNR. The mechanism responsible for the appearance of SR in this monostable system lies on the role 
played by noise in the relaxational dynamics itself towards the stable equilibrium state. As an important feature we have also found that the SNR depends on the amplitude of the oscillating field and reaches a maximum at a certain value of this parameter. This result reveals the existence of an optimal amplitude of the signal in order to be detected. Although the dipole is of interest in its own right, our results could also be extended to the situation in which the system is around an equilibrium state in a force field whose intensity varies periodically in time.

This work was supported by DGICYT of the Spanish Government under Grant No. PB92-0859. J.M.G.V. wishes to thank the Generalitat de Catalunya for financial support.
[1] R. Benzi, A. Sutera, and A. Vulpiani, J. Phys. A 14, L453 (1981).

[2] B. McNamara, K. Wiesenfeld, and R. Roy, Phys. Rev. Lett. 60, 2626 (1988).

[3] B. McNamara and K. Wiesenfeld, Phys. Rev. A 39, 4854 (1989).

[4] A. Simon and A. Libchaber, Phys. Rev. Lett. 68, 3375 (1992).

[5] Proceedings of the NATO Advanced Research Workshop on Stochastic Resonance, San Diego, 1992, edited by F. Moss, A. Bulsara, and M. F. Shlesinger [J. Stat. Phys. 70, 1 (1993)].

[6] F. Moss, in Some Problems in Statistical Physics, edited by G. H. Weiss (SIAM, Philadelphia, 1994).

[7] K. Wiesenfeld, D. Pierson, E. Pantazelou, C. Dames, and F. Moss, Phys. Rev. Lett. 72, 2125 (1994).

[8] K. Wiesenfeld and F. Moss, Nature 373, 33 (1995).

[9] Z. Gingl, L. B. Kiss, and F. Moss, Europhys. Lett. 29, 191 (1995).

[10] F. Marchesoni, L. Gammaitoni, and A. R. Bulsara, Phys. Rev. Lett. 76, 2609 (1996).

[11] S. Fauve and F. Heslot, Phys. Lett. 97A, 5 (1983).

[12] A. Longtin, A. Bulsara, and F. Moss, Phys. Rev. Lett. 67, 656 (1991).

[13] J. K. Douglass, L. Wilkens, E. Pantazelou, and F. Moss, Nature 365, 337 (1993).
[14] A. Peréz-Madrid and J.M. Rubí, Phys. Rev. E 51, 4159 (1995).

[15] Some authors, however, have found a maximum in the output signal for a monostable system. See, e.g., N. G. Stocks, N. D. Stein, and P. V. E. McClintock, J. Phys. A 26, L385 (1993).

[16] P. Debye, Polar Molecules (Chemical Catalog Company, New York, 1929).

[17] J. McConnell, Rotational Brownian Motion and Dielectric Theory (Academic, London, 1980).

[18] L. D. Landau and E. M. Lifshitz, Statistical Physics (Pergamon, New York, 1980), Vol. 5.

[19] W. T. Coffey, P. J. Cregg, and Yu P. Kalmykov, Adv. Chem. Phys. 83, 263 (1993).

[20] P. E. Kloeden and R. A. Pearson, J. Austral. Math. Soc. Ser. B 20, 8 (1977).

[21] J. R. Klauder and W. P. Petersen, SIAM J. Numer. Anal. 22, 1153 (1985).

[22] J. Iwaniszewski, Nuevo Cimento D 17, 819 (1995).

[23] C. W. Gardiner, Handbook of Stochastic Methods (SpringerVerlag, Berlin, 1990).

[24] M. I. Dykman, D. G. Luchinsky, R. Mannella, P. V. E. McClintock, N. D. Stein, and N. G. Stocks, Nuovo Cimento D 17, 661 (1995). 